\title{
Model-Based Structural Health Monitoring of Box Girders
}

\author{
Nicholas E. Silionis ${ }^{1}$, Konstantinos $N$. Anyfantis ${ }^{1, *}$, \\ ${ }^{1}$ Shipbuilding Technology Laboratory, School of Naval Architecture and Marine Engineering, \\ National Technical University of Athens, 106 82, Athens, Greece
}

\begin{abstract}
In the recent years, interest has been expressed towards incorporating Structural Health Monitoring (SHM) systems to ship hulls in order to transition from preventive to predictive maintenance procedures. In this work, an initial approach is undertaken to investigate the capabilities of a model-based method treating damage identification as an optimization problem solved using a genetic algorithm. An idealization of the hull structure is considered based on hull girder theory that allows for lab scale experimental testing. Specifically, a box girder is considered with a circular discontinuity as the generalized damage that causes extensive stress redistribution, replicating the effect of hull damage modes of interest. A three-point bending load case is considered to emulate still water bending loads. Damage is considered to exist, and the goal of the proposed strategy is to provide a prediction on its location and magnitude (level $2 \mathrm{SHM}$ ). This is achieved using strain measurements obtained from sensors located on theoretical zero-strain directions as inputs to the optimization scheme treating the damage identification problem. Results from both assessment strategies highlighted the influence of measurement-related uncertainties on the method's predictive capabilities.
\end{abstract}

\section{Introduction}

Maintenance procedures for ship structures are currently dictated by a set of rules and guidelines imposed by the International Maritime Organization (IMO), the International Association of Classification Societies (IACS) as well as individual classification societies. These are set around a strict schedule of visual inspections, known as surveys, where experts focus their attention on specific locations of the hull, which are considered susceptible to certain critical modes of damage [1]. Although this maintenance program has proven largely effective, due to its rigid nature it fails to offer real-time information on the structural condition of the hull structure. This constitutes a significant disadvantage for various facets of vessel operation. In the worst-case scenario, it may fail to provide warning on possible damages which may cause catastrophic collapse (e.g., MOL Comfort accident [2]), while in general it does not allow for the optimal planning of hull maintenance.

The above highlight that the integration of Structural Health Monitoring (SHM) systems for ship hulls can provide a viable solution to these shortcomings, something which is echoed

* Corresponding author: kanyf@,naval.ntua.gr 
by major classification societies (e.g., ABS) [3]. In general, SHM refers to systems which enable the automatic and on-line observation of the structural integrity of any given component or structure during service. Two main paradigms are recognized within this context, the so-called model-based and data-based [4]. In this work, the former is employed, following the example set by several works addressing SHM systems in the maritime sector [5-8]. More specifically, an idealized representation of the hull structure as a box-girder is theorized, following the example proposed by Anyfantis [9] and based on the principles of hull girder bending theory. To simulate still water sea-going and loading/ unloading (time invariant conditions) encountered during the ship's operational life, a three-point bending load case is considered. This choice furthermore enables an experimental assessment of the proposed SHM strategy, which utilizes an established combined FE-optimization scheme for damage identification [8], in which static strains are used as damage sensitive features, in the manner proposed by Preisler et. al. [10]. Ultimately, assessment is first performed on modelbased input data and then on data obtained from laboratory tests.

\section{Model-based SHM framework}

Ship structures are inherently highly complex, both in terms of their design configuration as well as their operating environment. Therefore, the use of an idealized model is justified in the context of a preliminary approach, such as the one considered herein.

\subsection{Idealized model description}

The idealized model of the hull structure chosen in this work is that of a square cross-section box girder. This is based primarily on two factors. First, on the fact that the global response of the hull structure is traditionally studied as the response of an equivalent prismatic beam, known as the hull girder [10]. And second, on the prospect of developing a model that is easily reproducible in an experimental environment, so as to enable lab-scale experiments. The three-point bending load case was selected based on the same considerations, as it is at the same time experimentally reproducible and leads to a longitudinal bending moment distribution across the girder adequately representing that caused by still water loading on actual vessels, as shown in Fig. 1. To justify this assumption, it was considered that only primary stresses act on the structure and localized phenomena were not considered.
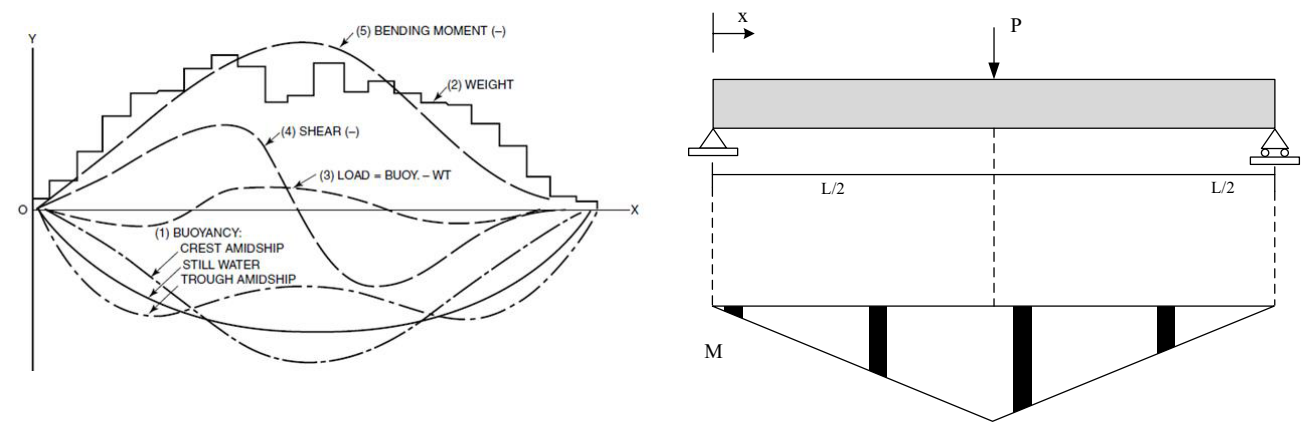

Fig. 1. Still-water bending moment distribution (left [12]) compared to three-point bending moment distribution (right)

As for the damage case, the design configuration and operational profile of ships is such that both the practical and regulatory approaches are more damage tolerant. Therefore, damages that cause significant stress redistribution are primarily of interest. Ultimately, it 
was decided to use a circular discontinuity (i.e., hole) as the idealization of the damage feature, which can also be easily implemented experimentally.

\subsection{Physics-based model}

The idealized box girder geometry was modelled using Finite Element procedures, in order to construct the physics-based model at the core of the proposed SHM framework. Quadrilateral linear shell elements were used for its discretization. A uniform, structured mesh was adopted with an element size capable of accurately capturing the disturbance caused on the strain field. Initially, a detailed model of the experimental set-up was developed using contact elements to model the interactions between the girder and the rollers, using a non-linear solution framework. However, as this model proved computationally costly, a simpler model was developed, solved solely within a linear framework, to assess its capacity as a fast and accurate alternative. Fig. 2 provides a comparison of the accuracy achieved by each model by plotting the longitudinal maximum stress distribution normalized with respect to its theoretical equivalent.

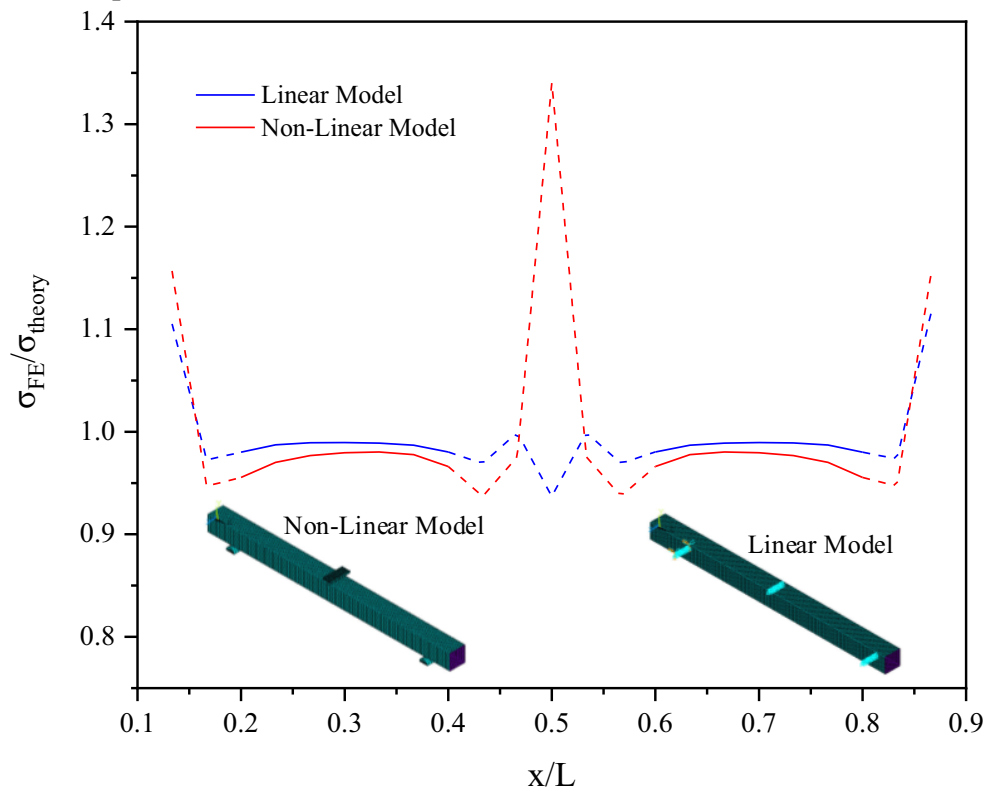

Fig. 2. Comparison of achieved accuracy between linear and non-linear models.

Evidently, the two models are equivalently accurate, both when compared to each other as well as the expected theoretical result, apart from areas near the rollers where local phenomena become prevalent. The linear model proved to be around 80 times faster, thus making it the choice moving forward.

\subsection{Damage sensitive features}

The damage sensitive feature used herein is strain measured on zero-strain directions of the undamaged girder, as specified by bending theory. These are: axial strain along the neutral axis of the webs and in-plane shear strain along the centerline of the flanges. Fig. 3 shows the sensor locations on these strain-monitoring paths, which are constrained, in terms of extent, to those regions were the contact effects have subsided, and thus the zero-strain baseline is not jeopardized. 


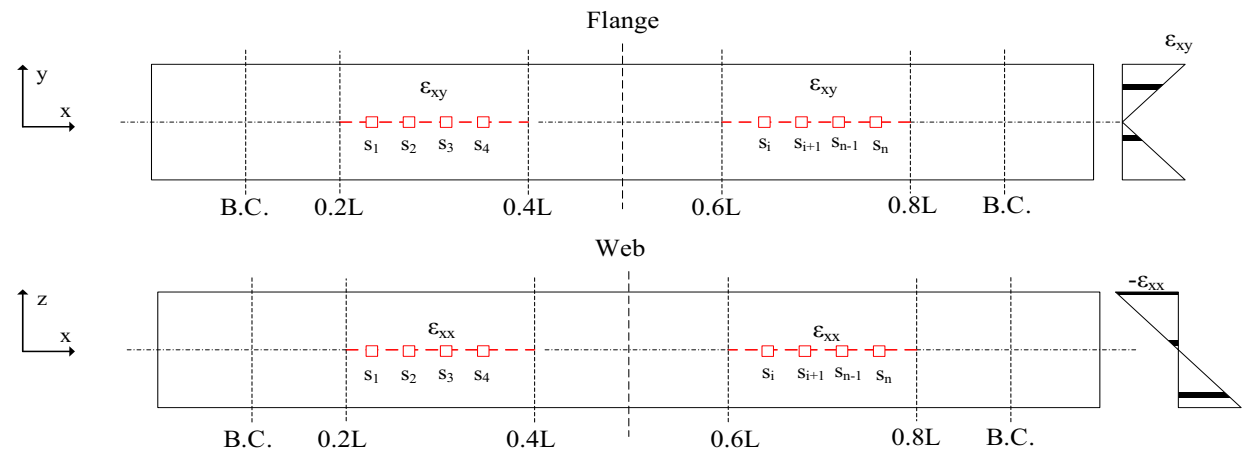

Fig. 3. Sensor locations along zero-strain monitoring paths for the girder's flanges and webs

\subsection{Damage identification strategy}

The goal of the proposed SHM framework is to provide a prediction on the location and magnitude of an already existing damage feature. This is achieved by treating damage identification as an optimization problem. An overview of the workflow of the combined SHM-optimization framework is given in Fig. 4 (a).

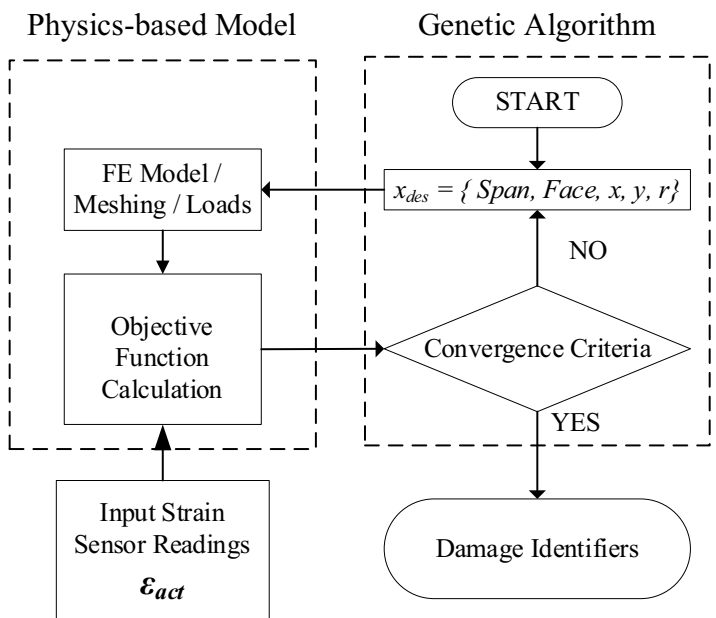

(a)

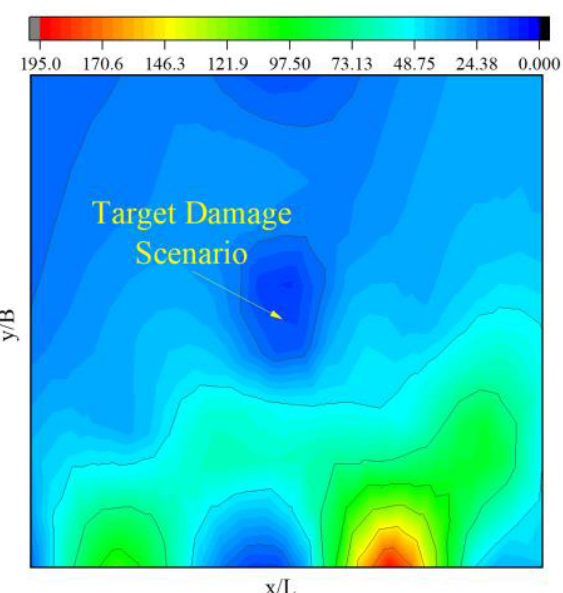

(b)

Fig. 4. (a) Workflow of combined FE - GA SHM framework (b) Non-linear objective function space

The design variables are the span (right or left of center) indication, face indication, location on each face and hole radius. In the approach considered herein, these variables are assumed as discrete and are subjected to constraints imposed by modelling parameters. The objective function is defined as follows, for each strain monitoring path:

$$
\left\|\varepsilon_{a c t}-\varepsilon_{v i r}(x)\right\|_{2}=\sqrt{\sum_{j=1}^{k}\left(\varepsilon_{a c t}-\varepsilon_{v i r}(x)\right)^{2}}
$$

Where $\varepsilon_{a c t}$ refers to the input strain measurements from the sensors on the structure and $\varepsilon_{v i r}(x)$ refers to strains obtained from the same locations, this time from the solution of the FE model for a set of input parameters $\boldsymbol{x}$ corresponding to a trial damage scenario generated by the optimizer. The objective function is an $\mathrm{L}_{2}$ norm between the input and trial strain vectors, chosen because its minimization comes when the two become near equal, thus leading to a 
solution which constitutes a prediction of the parameters of damage that caused the input measurements. This is evident in Fig. 4 (b), showing a contour plot of the objective function over a specific monitoring path, where it is minimized in the vicinity of a target damage scenario. The optimizer chosen in this work was a Multi-Objective Genetic Algorithm (MOGA), due in part to the discrete nature of the design variables as well as the highly nonlinear design space of the objective function (see Fig.4 (b)). The employed algorithm's convergence criteria were a Pareto percentage and a convergence stability criterion.

\section{Numerical assessment}

Initially the problem was solely assessed within a numerical environment. To obtain the required inputs, the FE model was solved for an indicative number of damage cases, representatively covering the flange and web regions. To assess the effectiveness of the proposed method on varying measurement information quantities, three virtual strain sensor configurations were used, each containing 5,10 and 16 virtual sensors per strain monitoring path. The number of sensors was selected to represent a decrease in the available measurement information. In order to emulate measurement-related uncertainty, an added noise component was introduced to the input strain data, drawn from a uniform distribution.

The predictions (i.e., design variables) obtained from the solution of the optimization problem, for the various damage cases and sensor configurations, were assessed in terms of their accuracy. In Fig. 5, a bar chart showing the maximum achievable accuracy of the proposed method is shown.

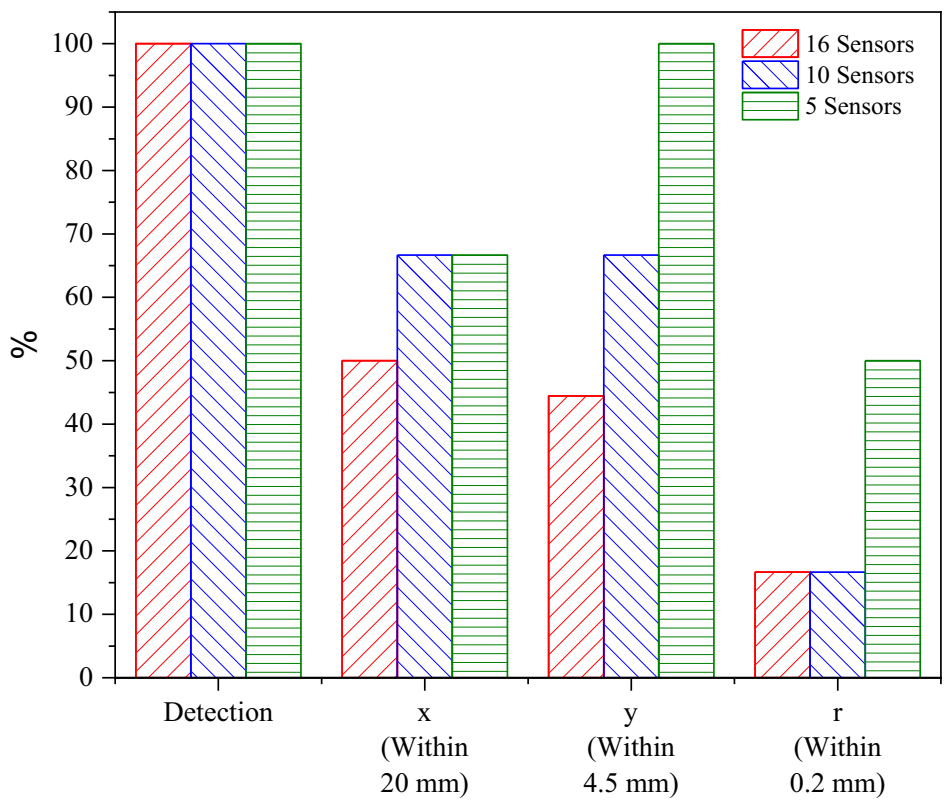

Fig. 5. Maximum achievable accuracy during the numerical assessment of the SHM framework

The accuracy terms referred to in Fig. 5, are defined as follows. First, a detection stage was defined based on the predicted span and face indication values. If both are correctly predicted by the algorithm, then damage is considered as detected. The next step follows only cases which were correctly detected and amounts to a prediction of the location of the damage, expressed by its coordinates on the local system defined for each face. This time, for a prediction to be counted as successful, it has to fall within a predefined region around the actual damage case, which spans 8 elements lengthwise and 2 elements width wise. 
Finally, to assign a successful count to the magnitude prediction, the hole radius obtained from the algorithm must fall within $1 / 10$ of the length of the interval of defined radius values.

The results provided in Fig. 5 correspond to the maximum added noise levels within which this accuracy is maintained consistently. These are provided in Table 1. Evidently, the smallest number of available measurements proved to be the most susceptible to noise influence, while at the same time achieving the highest performance. The latter is attributed to the superior performance of the GA in the lower-dimensional objective function space. In general, regardless of the number of virtual sensors, as added noise levels were increased a concurrent decrease in accuracy was observed. Finally, it was observed that damage cases located on the flange regions are overall more susceptible, due to the lower intensity in the in-plane shear strain field disturbance.

Table 1. Added noise range where accuracy is maintained for different virtual sensor numbers

\begin{tabular}{ccc}
\hline Virtual Sensor No. & \multicolumn{2}{c}{ Noise Range $[\mu \varepsilon]$} \\
\hline 5 sensors per path & Flange Cases & Web Cases \\
\hline 10 sensors per path & {$[-0.5,0.5]$} & {$[-1.5,1.5]$} \\
16 sensors per path & {$[-0.5,0.5]$} & {$[-2,2]$} \\
& {$[-2,2]$} & {$[-3,3]$} \\
\hline
\end{tabular}

\section{Experimental assessment}

For the experimental assessment of the proposed SHM strategy, a three point-bending test was performed at an MTS universal testing machine. In total, two box girder specimens were used, and four experiments were carried out. Each specimen was fitted with eight rosette strain gages, as shown in Fig. 6.

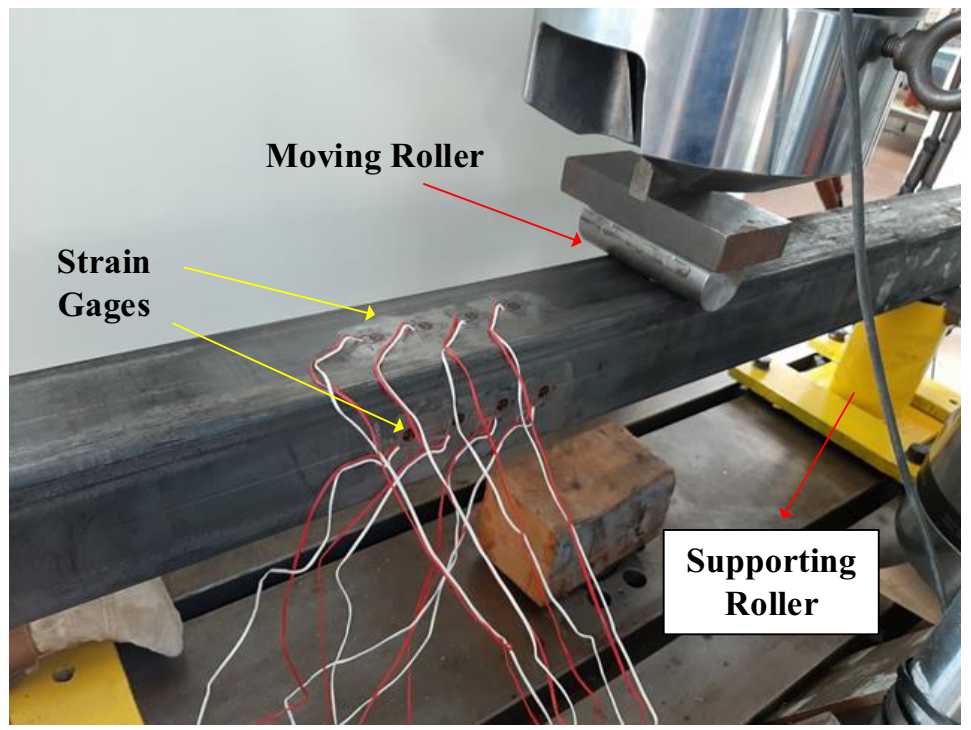

Fig. 6. Overview of experimental set-up used for the assessment of the SHM framework.

Two of the eight total strain monitoring paths (one flange and one web) were used in the experiments. Sensors were aligned close to each other to capture the localized effect of the damage-induced strain disturbance and close to the loading roller so that the higher induced strains would help drown out measurement noise. Also, the midship region of a ship is most 
susceptible to failure events and it was thus aimed to illustrate this importance in the experimental sensor alignment. The load was gradually applied to the pristine specimen until a prescribed force level. In load hold condition the drilling process began, and the hole was first opened at $3.5 \mathrm{~mm}$ diameter. Then, the specimen was left undisturbed for the vibrations to subside, and a quasi-static measurement was obtained, by averaging the strain results over the prescribed interval. This process was repeated for gradually increasing hole diameters up to $10 \mathrm{~mm}$. In Fig. 7 (a), an indicative strain history is shown, where drilling events are captured as spikes. The prescribed temporal intervals are annotated using dashed lines and roman numerals (I-VI). The results obtained from this process are shown in Fig. 7 (b), where it is evident that the damage is captured by the two sensors nearest to it. Also of note is that for the pristine specimen a non-zero baseline is registered, due to various factors (e.g., residual stresses, environmental factors etc.).

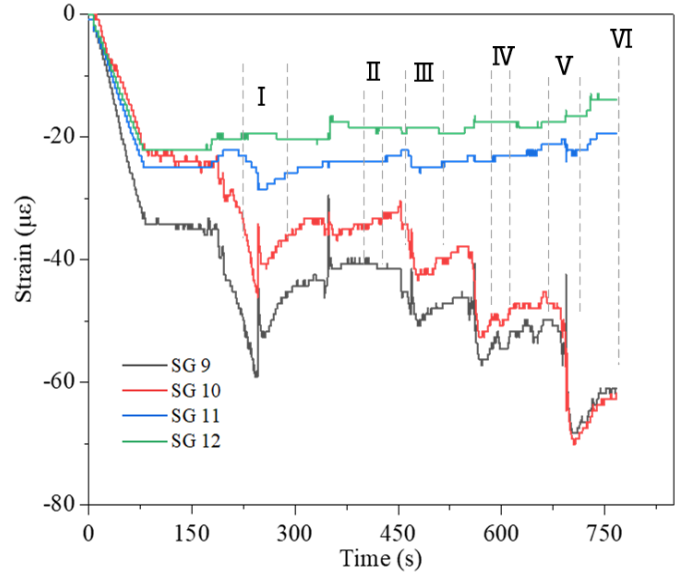

(a)

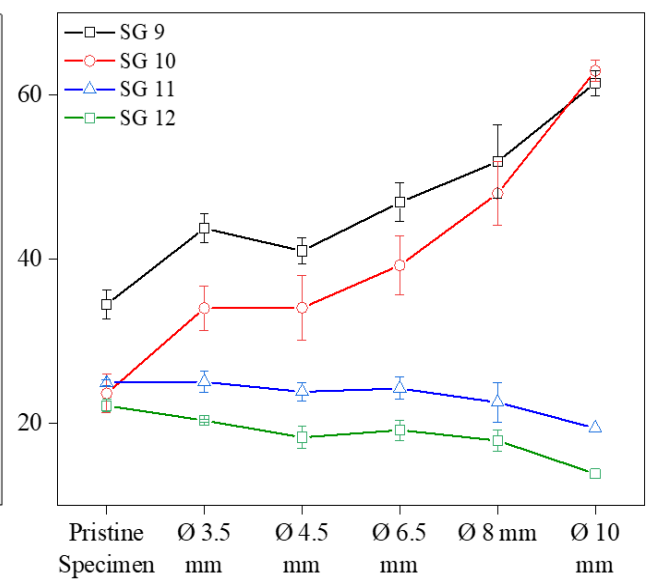

(b)

Fig. 7. (a) Recorded strain history during experiment (b) Corresponding static strain input data

A two-stage implementation was employed for the assessment on experimental data. In the first stage, a scaled down implementation was considered where the physics-based model was modified to replicate the experimental strain sensor alignment. It was expected that the low-dimensional input vector would increase the probability of accurate predictions. This would allow for the strongest candidate cases to be identified, to be then used during the second stage. Ultimately, in $2 / 4$ cases the detection stage was successful. Location predictions were highly accurate for one case (within one element difference), while for the other accuracy was significantly reduced. Both corresponded to the damage locations closest to the loading roller. Finally, it is worth mentioning that the assessment was performed for a given hole radius, and therefore no such prediction was made.

The two successfully predicted cases were then used for full-scale implementation. In that, the physics-based model contained the maximum number of sensors considered herein, i.e., 16 per strain monitoring path. To construct the input data vector, a synthesis of model and experimental data was performed. Namely, test measurements were introduced at their actual locations and for all other sensors, corresponding FE strains were used along with an added noise component, drawn from a uniform distribution within a range consistent with experimental data. When introduced to the SHM framework, the resulting predictions failed at the detection stage thus disqualifying the solutions. The opinion of the authors is that this is primarily a result of the significant influence of added noise in the employed highdimensional objective function. 


\section{Concluding remarks}

Overall, the monitoring strategy behind the model-based SHM framework proposed herein proved successful in capturing the presence of damage, both in the numerical as well as the experimental domain. The optimization-based strategy employed to identify an existing damage showed promise when assessed in a numerical environment but was proven to have limited accuracy on experimental input data. The influence of measurement and environment related uncertainties were identified as the key contributors to the limited achieved accuracy, especially when dealing with high-dimensional input data. Finding a way to mitigate this influence and further explore this research direction does, in our opinion, merit future investigation and is thus in the scope of our future research plans.

\section{References}

1. International Association of Classification Societies, No. 76 Guidelines for Assessment and Repair of Hull Structures - Bulk Carriers, (2007, last accessed 24 June 2021) https://www.iacs.org.uk/download/1863

2. Committee on Large Ship Container Safety Japan, Interim Report of Committee on Large Container Ship Safety, (2013, last accessed 25 June 2021) https://www.rina.org.uk/mol_comfort_accident.html

3. American Bureau of Shipping, Advisory on Structural Health Monitoring: The application of sensor-based approaches (2020, last accessed May 2021) https://ww2.eagle.org/content/dam/eagle/advisories-and-debriefs/abs-advisory-onstructural-health-monitoring-the-application-of-sensor-based-approaches.pdf

4. C. Farrar, K. Worden, Structural Health Monitoring: A Machine Learning Perspective (John Wiley \& Sons, Ltd, 2013)

5. A. Kefal, E. Oterkus, Displacement and stress monitoring of a chemical tanker based on inverse finite element method, Ocean Eng., 112, 33 (2016)

6. A. Kefal, E. Oterkus, Displacement and stress monitoring of a Panamax containership using inverse finite element method, Ocean Eng., 119, 19 (2016)

7. A Kefal et al, Three-dimensional shape and stress monitoring of bulk carriers based on iFEM methodology, Ocean Eng., 147, 256 (2018)

8. C. Earl, C. Stull, P.S. Koutsourelakis, Model-based Structural Health Monitoring of Naval Ship Hulls, Comput. Methods Appl. Mech Eng., 200, 1137 (2011)

9. K.N. Anyfantis, An abstract approach toward the structural digital twin of ship hulls: A numerical study applied to a box girder geometry, P I MECH ENG M-J ENG. (2021), doi:10.1177/1475090221989188.

10. Preisler A, Schröder KU, Schagerl M. Intrinsic Damage Assessment of Beam Structures Based on Structural Damage Indicators. Am J Eng Res 2018; 7(6): 56-70.

11. T. Yao, M. Fujikubo, Buckling and Ultimate Strength of Ship and Ship-like Floating Structures (Butterworth-Heinemann, 2016)

12. O.F. Hughes, J.K. Paik, Ship Structural Analysis and Design (The Society of Naval Architects and Marine Engineers, 2010) 